\title{
FIVE YEARS OF MASS DAPSONE (IDS) TREATMENT
}

A. S. Garrett, M.B., M.R.C.S., L.R.C.P. Area Superintendent, Onitsha Province, E. Nigeria

After five years. of treatment of large numbers of leprosy patients with oral Dapsone, I feel that a fair assessment of its advantages and disadvantages can be made. Of its success in reducing the total incidence of leprosy little will be said as Dr. T. F. Davey with the help of others, including myself, is preparing a paper about this.

Suffice it to say that total numbers reached, in Onitsha Province, were 10,600 in I95I when the use of Dapsone treatment had been fully launched (about a year after it began). Since then a steady decrease has taken place in total numbers to 7,800 at present. This has been due to a continued higher rate of discharge than new and relapsed cases added together. In addition the more severe types are seldom seen except in areas opened up recently.

\section{Progress through Treatment of Lepromatous Cases}

These have responded well bacteriologically. Out of over a thousand in various stages, there has been no exception unless some complication such a dermatitis has interfered with treatment. Nodules and infiltrations have become small, leaving skin wrinkled. Ears have sometimes remained thickened or misshapen, but signs of activity have gone.

The severest nodular lepromas, started on treatment in 1950, are now mostly reaching the stage of negative smears. All have much improved. In most the bacilli are much fragmented or have been reduced to acid-fast dust. The milder lepromas responded more quickly.

In I952, I7 of the more obstinate lepromas were given isoniazid in addition to dapsone. Progress was not much accelerated, but reaction seemed less. These and a few other patients had had sulphetrone since 1949.

\section{Complications}

After five years a better assessment can be made of complications than was made in the two preliminary articles in LEPROSY REvirw, July I95I and July I952. The incidence and importance 
of different complications seems to have changed, and certain original misconceptions have been proved false.

Dermatitis, with associated hepatitis, is by far the most severe and common of the complications caused by taking dapsone (as distinct from reactions of leprosy during treatment). The incidence of dermatitis continues to be low-about 2 per cent. Nearly all are fairly easily desensitised by a course of sulphetrone mixture starting with $\mathrm{I} / 8 \mathrm{o}$ gram. of sulphetrone. There remain a few who have recurrences. These, at first, were put on thiacetazone as an alternative method of treatment. As, however, it is now established that nearly all acquire a resistance to thiacetazone in about two years, we feel that this is not an adequate alternative.

We now give thiacetazone, but at the same time start a very slow and cautious desensitisation course, completing it in about six months instead of ten weeks with the normal course. This has proved successful.

Occasional cases of mental derangement continue to occur, but they are much rarer on twice-weekly treatment. Two mild cases have occurred in 680 settlement patients during the last year. This compares with 24 in the first year out of $\mathrm{I}$,ooo patients on daily treatment with dapsone. A few patients complain of sleeplessness or queer feelings in the head on treatment days. These cases are brought under control by reduction of dapsone dosage. The eariy recognition of such cases may, in part, be responsible for the low total numbers.

When dapsone was first used on a wide scale, we feared liver damage, jaundice, anaemia, ascites and oedema as complications of dapsone treatment. Dermatitis has, as part of its manifestation, a hepatitis which can be very severe, and if repeated can lead on to cirrhosis. Anaemia occurred in some cases and ascites and oedema are, of course, terminal events in both cirrhosis and anaemia of any cause. As a consequence great caution was at first used in the presence of either anaemia or enlarged liver. Extra iron was given as ferrous sulphate to all patients and any with signs of these diseases were given only very small doses of dapsone.

After five years of observation, I am convinced that this early caution was rather excessive. It is true that dermatitis, particularly if it is recurrent, causes severe hepatitis leading to cirrhosis, but in those who never show sign of dermatitis I have seen no evidence of liver damage. On the other hand, I have seen dozens (literally) of patients coming with large hard livers, obvious signs of undernourishment, anaemia and in some cases early oedema and ascites, who have greatly improved under treatment with dapsone. The liver has remained large, though in some cases a slight reduction in size has occurred. The other signs have improved and in some cases very greatly. Mild anaemia may occur in the first three or four months in a few cases, but soon disappears.

There are two explanations which I suggest for this. Dapsone, in many people, produces a much greater appetite. This may have a good tonic effect in undernourishment. It may, however, be noted that in this part of the country undernourishment is of quality rather than quantity of food, cassava taking a much greater place in the diet than it should. The other explanation may be that dapsone is a general bacteriostat and that some infection which is the hidden cause of the trouble may be overcome by it. Even many healthy patients have said that they are stronger and 
less easily tired when taking dapsone, though some complain of weakness on the day of treatment, particularly in the early months.

In fact, I believe that dapsone has a tonic action, but that the occasional sensitisation in which severe damage is done to the liver has led to it being regarded as a liver poison. Of course, as a general tonic, its occasional severe complications rule it out: Jaundice has practically never occurred since the early days. As there was an epidemic at that time affecting many without leprosy, I consider that this had no significance in relation to dapsone.

\section{Reactions}

Here it is important to state that we started with many severe lepromatous patients, some of whom had resisted hydnocarpus treatment for ten or more years. The new patients coming in now are more and more early cases of leprosy and therefore not subject to reactions in the same degree.

Lepra reaction (erythelila nodosum) occurs mainly in the severest lepromatous patients. As these cases are becoming rare, lepra reaction is met much less frequently than before. Treatment with Stibophen injections and reduction of dapsone normally control ihem within a few days. A few unfortunate patients have frequently repeated reactions, though most are now mild.

Eye reactions have become so rare that they need no special mention. Again they occurred in the most severe types of leproma in the early years of dapsone treatment.

Lepromatous nerve reactions are probably the most distressing complications of dapsone treatment for us now. They occur mostly at the time when bacteriological smears are becoming negative. They give rise in some cases to sometimes intractable pain and thickened tender nerves. I say " in some cases" advisedly. I have been surprised that many have had tender ulnar nerves as thick as a finger. They have had repeated acute pain in them. They have in some cases refused operation or had an operation on one arm but not on the other, equally affected. Yet a surprising number of them gradually improve with no loss of muscle power or feeling. This is at present a mystery. Others develop paralysis and wasting.

Injection of procaine gives temporary relief in early cases, but after a time the nerve is too hard to have anything injected into it. Incision with or without stripping of the sheath, relieves pain in most cases, but by no means all. At operation, it is often noted that the sheath is exceptionally hard and adherent even in those with little or no paralysis or wasting. After trial of early and late operation, injection with procaine, reduction or stoppage of dapsone, alternative treatment with thiacetazone and use of antihistamines, ACTH or sedatives, I think the best treatment is as follows:-

Reduction of dose temporarily (2-4 weeks to $\frac{1}{2}$ dose) relieves the reaction in many cases and often there is no serious recurrence, or if it occurs, it may be dealt with in the same way. In some cases, reactions occur with increasing severity and reduction of dose does not relieve the situation unless it is prolonged on a very low dosage level. In these cases 
it is often worth transferring to thiacetazone therapy which gives rise to fewer nerve reactions. Thiacetazone, however, is not always successful and at any rate the drug leads to resistance after about two years of use. Usually, however, this period gives a long enough respite and the reactions seldom recur when dapsone is restarted.

Sedatives such as Tab. Codeine Co. are very useful. Occasionally morphine or pethidine may be needed in severe cases. Operation with stripping or incision of the sheath would appear to be logical in a condition with such great swelling and tenderness. But the fact that many show none of the symptoms of pressure on the nerve fibres-paralysis and anaesthesia-even after repeated reactions, makes one wonder whether it is necessary unless one or other of these signs are appearing. The results are by no means consistently successful and pain may continue after the operation. If practised on early cases, the results are open to question, as many get better after one or a few reactions without paralysis or anaesthesia. My own practice is to operate on fewer lepromatous nerves than previously.

$\Lambda C$ 'TI has been used and it reduces the pain temporarily, but with its adverse effect on the general course of the disease, it is a type of treatment only very rarely to be considered. Exercises, reduction of dapsone and patience on the part of staff and patient seem to be the most important methods of overcoming this, the most distressing trouble arising during dapsone treatment of lepromas.

* An experiment with injection of hyalase and procaine has been started and gives good early promise. I hope to report more fully when I have had time to assess the results.

Tuberculoid nerve reactions do not occur frequently, but due to the caseation, they can cause severe and permanent damage in a short time. Early operation is the only remedy for this.

In the borderline and in the atypical tuberculoid leprosy, particularly if the lesions are much raised and situated on hands, feet or face, severe reactions with paralysis often occur early in treatment. This is particularly true if the dosage is rapidly raised. In these cases I now start treatment on a low dosage and increase more slowly, instructing the Leprosy Inspector administering the drug to observe carefully for nerve reactions and reduce or stop Dapsone at the first sign, referring the patient to the doctor.

Nur normal induction of treatment is:roo $\mathrm{mg}$. Dapsone twice weekly for 3 weeks $200 \mathrm{mg}$. ". ". ,. ,. , , , 300 mg. ". 400 mg. maximum." ". ", ., ",

For these cases, I usually advise a maximum of $200 \mathrm{mg}$. for at least three months. When reactions occur in this type, they are usually very different from those seen in leproma. Pain is not a marked feature, but severe paralysis and anaesthesia may occur early in the reaction. Stoppage of Dapsone does not lead to a rapid reduction of reaction or to recovery from paralysis. But with patience, exercises and careful raising of dapsone losage most of these patients gradually recover the use of their limbs.

Nerve stripping may be useful, but I have found many with such a length of nerve thickened that operation would be impracticable. An example of this is in hospital now with ulnars, medians and medial antebrachial cutaneous nerves greatly thickened from the axilla lown to the lowest branches. The radials are also affected, but cannot be so readily felt. Paralysis appears almost complete, but I have seen such cases recover a considerable amount of movement.

\footnotetext{
"See the next article.
} 
About a quarter of the tuberculoid and indeterminate cases at some tine during their treatment have crops of new macules, but unless they are accompanied by nerve signs it seems better to continue them on full closage of Ilapsone.

\section{Residual Hypopigmentation}

This is distressing to the patient who has, after all, come for treatment of the unsightly patches on his skin which make his friends and relatives shun his company. If he returns home after treatment with much hypopigmentation it is difficult for his home people to feel that the leprosy is properly cured.

Hypopigmentation also poses a problem to the doctor responsible for discharging him as free from active leprosy and perhaps still more for the person re-examining discharged patients. An over-anxious patient, or one whose relatives are worried, will often claim as new a patch which has been present for years. Many times even birthmarks have been produced as new patches of leprosy to prove the necessity to continue or restart treatment.

We have found it useful to estimate the percentage hypopigmentation and not to regard it as inactive until it remains constant for two or three examinations. This is obviously only a rough guide, as different observers, different light and perhaps different opinion by the same person all have their effect.

In inactive patches it is often found that the hypopigmentation is irregular, with greater loss of colour in the wrinkles of the skin than in the raised parts. In some cases there is apparent thickening of the skin, but on palpation, stretching and pinching, it is found that it is only due to course wrinkling from loss of elasticity.

When hypopigmentation has become stationary and there is no sign of erjthema or thickening of the macule, trichloracetic acid 25 per cent, seems to be as satisfactory as any. That or any other caustic tends to form a dark scar, sometimes mottled, but it satisfies the patient and his relatives and is therefore justified. Overcautious application is practically useless.

\section{Drug Resistance}

No definite case of Dapsone resistance has yet been shown by us but we have a few cases which lead us to suspect its development. One lepromatous patient has shown slow but steady improvement over five years of Sulphetrone and Dapsone treatment, but in the last two smear results there is a slight rise again. No clinical deterioration is shown and the bacteriological index is still well below that at the start of treatment. A few tuberculoid patients remain with active-looking lesions for four or more years. These cases must be watched and if the drug-resistance is confirmed it will be reported.

\section{Relapses}

During the past three and a half years we have been averaging nearly 3,000 discharged per annum. Few of these have been lepromatous except in recent times.

The average relapse rate is about 7 per cent.

This, however, does not give a true picture. Relapses have occurred mainly in three types of patient:- 
(a) In those previously treated with hydnocarpus oil and apparently inactive, many were given a course of dapsone of one year or less.

(b) In clinically tuberculoid cases, not completely typical, either with not very clearly defined edges or with lack of healing centre, response to treatment is usually dramatic. Lesions disappear in a few months, but lacking an initial positive smear, they were given a relatively short course of Dapsone, usually I8 months.

(c) Those not attending regularly for treatment due to laziness or personal difficulties.

\section{Criteria for Discharge}

In all cases a minimum of 24 months' treatment is now observed. In nearly all cases 30 or more months' treatment are given. Typical tuberculoid cases with few lesions and strongly healing centre are the least likely to relapse. Extensive lesions, poor central healing or poor definition call for at least $2 \frac{1}{2}$ to 3 years' treatment. When the smear has been positive, at least 2 years' treatment is given after the first negative smear. In cases where the patient is content to stay on, this is prolonged.

\section{Organisation}

We have had two doctors most of the time-sometimes only one-and two Leprosy Control Officers, laymen with teaching in leprosy who are responsible for clinical supervision, supplies, sanitation and the many problems which arise in district work. The actual administration of Dapsone and dealing with the complications in the first instance, are in the hands of the Leprosy Inspectors, some with primary and some with secondary education plus six months to a year of tuition by us. There are 56 clinics and about 7,800 patients at present.

This organisation is quite adequate for the simple cases. The more difficult patients are brought in to the Settlement or to the hospital, which has a trained nursing staff plus ward attendants who are themselves patients. This enables a closer watch to be kept on those needing most attention.

\section{Safety of Mass Dapsone as Against Alternatives}

At the commencement of treatment by this method the obvious dangers of Dapsone-dermatitis, psychosis, severe reactions and occasional death arising from the first two were pointed out. Now an assessment of the alternatives can be made. With the adoption of twice-weekly treatment, and with experience in treatment of complication, the dangers are much less than they seemed at first.

Hydnocarpus oil was much less successful in treatment, but had less dramatic and obvious complications. Cases did, however, occur of tetanus, and I believe death from that cause was greater than death from Dapsone, even in the beginning. 
Injections of Dapsone in oil or Sulphetrone carry this danger when administered by relatively untrained staff. They also involve greater organisational difficulties. Provision of a fully responsible trained staff with all facilities is impossible owing to lack of qualified people and of funds.

The only real alternatives, therefore, are to provide sulphone treatment in some way similar to that described, or not to provide anything for the majority of sufferers. This would, and does, from actual experience, result in black market sale of Dapsone with all the dangers inherent in the system described, plus others such as great overdose by enthusiastic salesmen or persistent underdose and irregular treatment for those who cannot afford it.

The dangers and advantages of injections of Dapsone in oil or sulphetrone as against tablets might be weighed up according to the local conditions of population density, roads, leprosy incidence and the staff available.

Abuses. I think it necessary to mention some abuses to which this drug is put, the cure of which is a moral and social rather than a medical problem. There is a wide illicit trade in dapsone as in all successful medicines in this country. Precautions, as much as we are able, are taken by us to avoid theft. Import restrictions are made, but still the trade goes on.

The knowledge that such trade exists makes us speak hesitantly about real statistics, though the fact that an ever-decreasing number of severe cases present themselves shows that the trend towards extinction of leprosy is really present.

The real danger to the whole campaign, I believe, lies in the early lepromatous patient who can casily conceal his disease by treatment which is insufficient to make him uninfective. Will this produce a drug-resistant strain? Will the hidden infecting agents be sufficient to keep leprosy control always in the future. Time alone will tell.

Meanwhile propaganda is a vital weapon, by written and spoken word and we hope a recently produced film will be really helpful.

\section{Summary}

Five years of experience in widespread oral Dapsone treatment has shown its practicability with a small qualified staff and a larger locally trained staff.

The difficulties arising from drug sensitivity and reactions of the disease, together with their treatment are discussed. So also are the organisational difficulties. 